\title{
Correction to: Patterns and controls of mercury accumulation in sediments from three thermokarst lakes on the Arctic Coastal Plain of Alaska
}

\author{
S. M. Burke' ${ }^{1}$ C. E. Zimmerman ${ }^{2}$ - B. A. Branfireun ${ }^{3}$ - J. C. Koch ${ }^{2} \cdot$ H. K. Swanson ${ }^{1}$
}

Published online: 7 March 2020

(c) Springer Nature Switzerland AG 2020

\section{Correction to: Aquatic Sciences (2018) 80:1 https://doi.org/10.1007/s00027-017-0553-0}

The following corrections pertain to a calculation error associated with sediment focusing factors.

Page 1, Abstract, lines 6-8: The following sentence, which previously read:

"Mercury accumulation in two of the three lakes was variable and high over the past century (91.96 and $78.6 \mu \mathrm{g} / \mathrm{m}^{2} /$ year), and largely controlled by sedimentation rate. Mercury accumulation in the third lake was lower $\left(14.2 \mu \mathrm{g} / \mathrm{m}^{2} / \mathrm{year}\right)$, more temporally uniform, and was more strongly related to sediment $\mathrm{Hg}$ concentration than sedimentation rate."

should read:

"Mercury accumulation in two of the three lakes was variable and high over the past century (37.4 and $45.84 \mu \mathrm{g} / \mathrm{m}^{2} /$ year), and largely controlled by sedimen-

The original article can be found online at https://doi.org/10.1007/ s00027-017-0553-0.

Electronic supplementary material The online version of this article (https://doi.org/10.1007/s00027-020-0707-3) contains supplementary material, which is available to authorized users.

H. K. Swanson

heidi.swanson@uwaterloo.ca

S. M. Burke

samantha.burke2@gmail.com

1 Department of Biology, University of Waterloo, Waterloo, ON, Canada

2 US Geological Survey, Alaska Science Center, Anchorage, AK, USA

3 Department of Biology and Centre for Environment and Sustainability, University of Western Ontario, London, Canada tation rate. Mercury accumulation in the third lake was lower $\left(6.46 \mu \mathrm{g} / \mathrm{m}^{2} /\right.$ year $)$, more temporally uniform, and was more strongly related to sediment $\mathrm{Hg}$ concentration than sedimentation rate."

Page 5, Table 1: The following table which previously read:

Table 1 Physical characteristics and summary $\mathrm{Hg}$ accumulation results of the three study lakes (BRW100, ATQ206, RDC312) on the Arctic Coastal Plain of Alaska; $\mathrm{Hg}$ accumulation trends were assessed using Mann-Kendall tests (a p value $<0.05$ denotes a significant temporal trend and the Kendall's tau denotes the direction and magnitude of the relationship); McLeod (2011); catchments were delineated, and percent growth was calculated using ArcMap ${ }^{\mathrm{TM}}$ 10.2.2 (ESRI 2016)

\begin{tabular}{|c|c|c|c|}
\hline & BRW100 & ATQ206 & RDC312 \\
\hline $\begin{array}{l}\text { Latitude (decimal } \\
\text { degrees) }\end{array}$ & 71.24163 & 70.41557 & 69.95348 \\
\hline $\begin{array}{l}\text { Longitude (decimal } \\
\text { degrees) }\end{array}$ & -156.77391 & -156.98128 & -156.63817 \\
\hline Surface area $\left(\mathrm{km}^{2}\right)$ & 1.7 & 1.8 & 0.7 \\
\hline $\begin{array}{l}\text { Catchment area } \\
\left(\mathrm{km}^{2}\right)\end{array}$ & 24.0 & 22.8 & 29.9 \\
\hline $\begin{array}{l}\text { Catchment to sur- } \\
\text { face area ratio }\end{array}$ & 13.7 & 12.8 & 41.0 \\
\hline $\begin{array}{l}\text { Growth since } 1948 \\
(\%)\end{array}$ & 12.7 & -0.3 & 5.4 \\
\hline Landscape type & $\begin{array}{l}\text { Lake thermo- } \\
\text { karst }\end{array}$ & $\begin{array}{l}\text { Lake thermo- } \\
\text { karst }\end{array}$ & $\begin{array}{l}\text { Lake thermo- } \\
\text { karst }\end{array}$ \\
\hline $\begin{array}{l}\text { Mean } \mathrm{Hg} \text { accumula- } \\
\text { tion }\left(\mu \mathrm{g} / \mathrm{m}^{2} / \text { year }\right)\end{array}$ & 92.0 & 14.2 & 78.7 \\
\hline Standard deviation & 36.1 & 3.6 & 68.5 \\
\hline $\begin{array}{l}\mathrm{Hg} \text { accumulation } \\
\text { trend }\end{array}$ & No trend & Positive & No trend \\
\hline Kendall's tau $(\tau)$ & 0.28 & 0.42 & 0.61 \\
\hline $\begin{array}{l}\text { Mann-Kendall p } \\
\text { value }\end{array}$ & 0.17 & 0.04 & 0.84 \\
\hline
\end{tabular}

Should read: 
Table 1 Physical characteristics and summary $\mathrm{Hg}$ accumulation results of the three study lakes (BRW100, ATQ206, RDC312) on the Arctic Coastal Plain of Alaska; $\mathrm{Hg}$ accumulation trends were assessed using Mann-Kendall tests (a p value $<0.05$ denotes a significant temporal trend and the Kendall's tau denotes the direction and magnitude of the relationship); McLeod (2011); catchments were delineated, and percent growth was calculated using ArcMap ${ }^{\mathrm{TM}}$ 10.2.2 (ESRI 2016)

\begin{tabular}{|c|c|c|c|}
\hline & BRW100 & ATQ206 & RDC312 \\
\hline $\begin{array}{l}\text { Latitude (decimal } \\
\text { degrees) }\end{array}$ & 71.24163 & 70.41557 & 69.95348 \\
\hline $\begin{array}{l}\text { Longitude (deci- } \\
\text { mal degrees) }\end{array}$ & -156.77391 & -156.98128 & -156.63817 \\
\hline $\begin{array}{l}\text { Surface area } \\
\left(\mathrm{km}^{2}\right)\end{array}$ & 1.7 & 1.8 & 0.7 \\
\hline $\begin{array}{l}\text { Catchment area } \\
\left(\mathrm{km}^{2}\right)\end{array}$ & 24.0 & 22.8 & 29.9 \\
\hline $\begin{array}{l}\text { Catchment to sur- } \\
\text { face area ratio }\end{array}$ & 13.7 & 12.8 & 41.0 \\
\hline $\begin{array}{c}\text { Growth since } \\
1948(\%)\end{array}$ & 12.7 & -0.3 & 5.4 \\
\hline Landscape type & $\begin{array}{l}\text { Lake thermo- } \\
\text { karst }\end{array}$ & $\begin{array}{l}\text { Lake thermo- } \\
\text { karst }\end{array}$ & $\begin{array}{l}\text { Lake thermo- } \\
\text { karst }\end{array}$ \\
\hline $\begin{array}{l}\text { Mean } \mathrm{Hg} \text { accumu- } \\
\text { lation }\left(\mu \mathrm{g} / \mathrm{m}^{2} /\right. \\
\text { year) }\end{array}$ & 37.4 & 6.5 & 45.8 \\
\hline $\begin{array}{l}\text { Standard devia- } \\
\text { tion }\end{array}$ & 14.7 & 1.6 & 40.5 \\
\hline $\begin{array}{l}\mathrm{Hg} \text { accumulation } \\
\text { trend }\end{array}$ & No trend & Positive & No trend \\
\hline Kendall's tau $(\tau)$ & 0.28 & 0.42 & 0.61 \\
\hline $\begin{array}{l}\text { Mann-Kendall p } \\
\text { value }\end{array}$ & 0.17 & 0.04 & 0.84 \\
\hline
\end{tabular}

Page 6, Table 2: The following Table which previously read:

Table 2 Mean $\mathrm{Hg}$ accumulation and Mann-Kendall results for thirtyseven previously studied Arctic and subarctic lakes; landscape types were designated using the maps generated by Olefeldt et al. 2016; further lake information available in the Supplemental Materials

\begin{tabular}{|c|c|c|c|c|c|c|c|}
\hline Lake & Country & $\begin{array}{l}\text { Landscape } \\
\text { type }\end{array}$ & $\begin{array}{l}\text { Mean } \mathrm{Hg} \\
\text { Accum } \\
\left(\mu \mathrm{g} / \mathrm{m}^{2} / \text { year }\right)\end{array}$ & $\sigma$ & Trend & $\tau$ & $\mathrm{p}$ \\
\hline $2-A$ & Canada & Lake & 62.23 & 18.04 & neg & $0.71^{-}$ & 0.019 \\
\hline $2-B$ & Canada & Lake & 23.88 & 13.58 & pos & 0.91 & 0.007 \\
\hline Amituk & Canada & $\begin{array}{l}\text { Non- } \\
\text { thermo- } \\
\text { karst }\end{array}$ & 17.98 & 7.48 & pos & 0.70 & 0.02 \\
\hline AX-AJ & Canada & Hillslope & 9.85 & 4.17 & pos & 0.94 & $<0.001$ \\
\hline BI-02 & Canada & Hillslope & 4.94 & 1.40 & pos & 0.39 & 0.047 \\
\hline BK-AH & Canada & Hillslope & 4.54 & 0.98 & $\mathrm{n} / \mathrm{a}$ & $0.39^{-}$ & 0.062 \\
\hline Brady & USA & $\begin{array}{l}\text { Non- } \\
\text { thermo- } \\
\text { karst }\end{array}$ & 8.36 & 1.62 & pos & 0.46 & 0.033 \\
\hline Burial & USA & Hillslope & 4.40 & 1.12 & pos & 0.71 & 0.019 \\
\hline
\end{tabular}

\begin{tabular}{|c|c|c|c|c|c|c|c|}
\hline Lake & Country & $\begin{array}{l}\text { Landscape } \\
\text { type }\end{array}$ & $\begin{array}{l}\text { Mean Hg } \\
\text { Accum } \\
\left(\mu \mathrm{g} / \mathrm{m}^{2} / \text { year }\right)\end{array}$ & $\sigma$ & Trend & $\tau$ & $\mathrm{p}$ \\
\hline CF-11 & Canada & $\begin{array}{l}\text { Non- } \\
\text { thermo- } \\
\text { karst }\end{array}$ & 2.56 & 1.47 & pos & 1.00 & 0.009 \\
\hline Char & Canada & $\begin{array}{l}\text { Non- } \\
\text { thermo- } \\
\text { karst }\end{array}$ & 14.38 & 6.04 & pos & 0.89 & 0.0001 \\
\hline Daglet & USA & $\begin{array}{l}\text { Non- } \\
\text { thermo- } \\
\text { karst }\end{array}$ & 8.29 & 1.61 & pos & 0.78 & 0.002 \\
\hline Daltjørna & Norway & $\begin{array}{l}\text { Non- } \\
\text { thermo- } \\
\text { karst }\end{array}$ & 22.70 & 4.81 & pos & 0.78 & 0.0001 \\
\hline DV-E & Canada & Hillslope & 0.89 & 0.15 & $\mathrm{n} / \mathrm{a}$ & 0.67 & 0.308 \\
\hline Efficient & USA & Hillslope & 8.71 & 3.26 & pos & 0.64 & $<0.0001$ \\
\hline Forgetful & USA & Hillslope & 10.13 & 2.66 & pos & 0.49 & 0.013 \\
\hline Hazen & Canada & Hillslope & 31.55 & 3.30 & $\mathrm{n} / \mathrm{a}$ & $0.10^{-}$ & 0.442 \\
\hline Lake 53 & $\begin{array}{l}\text { Green- } \\
\text { land }\end{array}$ & $\begin{array}{l}\text { Non- } \\
\text { thermo- } \\
\text { karst }\end{array}$ & 3.69 & 1.32 & pos & 0.86 & $<0.0001$ \\
\hline Lake 70 & $\begin{array}{l}\text { Green- } \\
\text { land }\end{array}$ & $\begin{array}{l}\text { Non- } \\
\text { thermo- } \\
\text { karst }\end{array}$ & 6.23 & 3.00 & $\mathrm{n} / \mathrm{a}$ & 0.41 & 0.127 \\
\hline $\begin{array}{l}\text { Matacha- } \\
\text { rak }\end{array}$ & USA & $\begin{array}{l}\text { Non- } \\
\text { thermo- } \\
\text { karst }\end{array}$ & 3.64 & 1.52 & pos & 0.83 & 0.002 \\
\hline MB-AC & Canada & Hillslope & 6.15 & 1.25 & pos & 0.84 & $<0.0001$ \\
\hline MB-S & Canada & Hillslope & 2.44 & 0.14 & $\mathrm{n} / \mathrm{a}$ & 0.00 & 1 \\
\hline Mcleod & USA & Hillslope & 17.93 & 4.73 & pos & 0.48 & 0.006 \\
\hline North & Canada & Hillslope & 53.96 & 20.98 & pos & 0.82 & 0.0003 \\
\hline Nunatak & $\begin{array}{l}\text { Green- } \\
\text { land }\end{array}$ & $\begin{array}{l}\text { Non- } \\
\text { thermo- } \\
\text { karst }\end{array}$ & 8.24 & 1.62 & pos & 0.81 & 0.016 \\
\hline $\begin{array}{l}\text { Ossian } \\
\text { Sars- } \\
\text { fjellet }\end{array}$ & Norway & $\begin{array}{l}\text { Non- } \\
\text { thermo- } \\
\text { karst }\end{array}$ & 4.00 & 1.86 & pos & 0.72 & 0.009 \\
\hline Perfect & USA & Hillslope & 7.07 & 1.94 & pos & 0.88 & $<0.0001$ \\
\hline Relaxing & USA & Hillslope & 2.43 & 0.97 & pos & 0.85 & $<0.0001$ \\
\hline $\begin{array}{l}\text { Rocky } \\
\text { Basin }\end{array}$ & Canada & $\begin{array}{l}\text { Non- } \\
\text { thermo- } \\
\text { karst }\end{array}$ & 1.26 & 0.53 & pos & 1.00 & 0.03 \\
\hline Romulus & Canada & Hillslope & 198.19 & 67.70 & pos & 0.78 & 0.002 \\
\hline Rummy & Canada & Hillslope & 13.19 & 1.83 & $\mathrm{n} / \mathrm{a}$ & 0.62 & 0.072 \\
\hline SHI-L4 & Canada & $\begin{array}{l}\text { Non- } \\
\text { thermo- } \\
\text { karst }\end{array}$ & 20.00 & 9.39 & pos & 0.82 & $<0.0001$ \\
\hline SHI-L7 & Canada & $\begin{array}{l}\text { Non- } \\
\text { thermo- } \\
\text { karst }\end{array}$ & 2.64 & 0.73 & $\mathrm{n} / \mathrm{a}$ & 0.17 & 0.602 \\
\hline Surprise & USA & Hillslope & 20.98 & 11.47 & pos & 0.90 & $<0.0001$ \\
\hline Vassauga & Norway & $\begin{array}{l}\text { Non- } \\
\text { thermo- } \\
\text { karst }\end{array}$ & 7.30 & 3.35 & pos & 0.93 & 0.002 \\
\hline West & Canada & hillslope & 23.44 & 11.79 & pos & 0.76 & 0.006 \\
\hline Wonder & USA & hillslope & 31.50 & 15.31 & pos & 0.93 & 0.002 \\
\hline Yterjørna & Norway & $\begin{array}{l}\text { non- } \\
\text { thermo- } \\
\text { karst }\end{array}$ & 12.21 & 9.69 & pos & 0.84 & $<0.0001$ \\
\hline
\end{tabular}


Should read:

Table 2 Mean Hg accumulation and Mann-Kendall results for thirtyseven previously studied Arctic and subarctic lakes; landscape types were designated using the maps generated by Olefeldt et al. 2016; further lake information available in the Supplemental Materials

\begin{tabular}{|c|c|c|c|c|c|c|c|}
\hline Lake & Country & $\begin{array}{l}\text { Landscape } \\
\text { type }\end{array}$ & $\begin{array}{l}\text { Mean } \mathrm{Hg} \\
\text { Accum. } \\
\left(\mu \mathrm{g} / \mathrm{m}^{2} /\right. \\
\text { year })\end{array}$ & $\sigma$ & Trend & $\tau$ & $\mathrm{p}$ \\
\hline $2-\mathrm{A}$ & Canada & Lake & 62.23 & 18.04 & neg & -0.71 & 0.019 \\
\hline 2-B & Canada & Lake & 23.88 & 13.58 & pos & 0.91 & 0.007 \\
\hline Amituk & Canada & $\begin{array}{l}\text { Non- } \\
\text { thermo- } \\
\text { karst }\end{array}$ & 1.34 & 0.56 & pos & 0.70 & 0.02 \\
\hline AX-AJ & Canada & Hillslope & 32.55 & 13.79 & pos & 0.94 & $<0.001$ \\
\hline BI-02 & Canada & Hillslope & 6.53 & 1.86 & pos & 0.39 & 0.047 \\
\hline BK-AH & Canada & Hillslope & 2.73 & 0.59 & $\mathrm{n} / \mathrm{a}$ & -0.39 & 0.062 \\
\hline Brady & USA & $\begin{array}{l}\text { Non- } \\
\text { thermo- } \\
\text { karst }\end{array}$ & 11.84 & 2.30 & pos & 0.46 & 0.033 \\
\hline Burial & USA & Hillslope & 5.69 & 1.45 & pos & 0.71 & 0.019 \\
\hline CF-11 & Canada & $\begin{array}{l}\text { Non- } \\
\text { thermo- } \\
\text { karst }\end{array}$ & 1.07 & 0.62 & pos & 1.00 & 0.009 \\
\hline Char & Canada & $\begin{array}{l}\text { Non- } \\
\text { thermo- } \\
\text { karst }\end{array}$ & 1.49 & 0.62 & pos & 0.89 & 0.0001 \\
\hline Daglet & USA & $\begin{array}{l}\text { Non- } \\
\text { thermo- } \\
\text { karst }\end{array}$ & 10.23 & 1.99 & pos & 0.78 & 0.002 \\
\hline Daltjørna & Norway & $\begin{array}{l}\text { Non- } \\
\text { thermo- } \\
\text { karst }\end{array}$ & 22.70 & 4.81 & pos & 0.78 & 0.0001 \\
\hline DV-E & Canada & Hillslope & 4.02 & 0.66 & $\mathrm{n} / \mathrm{a}$ & 0.67 & 0.308 \\
\hline Efficient & USA & Hillslope & 2.44 & 0.91 & pos & 0.64 & $<0.0001$ \\
\hline Forgetful & USA & Hillslope & 3.16 & 0.83 & pos & 0.49 & 0.013 \\
\hline Hazen & Canada & Hillslope & 21.91 & 2.29 & $\mathrm{n} / \mathrm{a}$ & -0.10 & 0.442 \\
\hline Lake 53 & $\begin{array}{c}\text { Green- } \\
\text { land }\end{array}$ & $\begin{array}{l}\text { Non- } \\
\text { thermo- } \\
\text { karst }\end{array}$ & 3.69 & 1.32 & pos & 0.86 & $<0.0001$ \\
\hline Lake 70 & $\begin{array}{c}\text { Green- } \\
\text { land }\end{array}$ & $\begin{array}{l}\text { Non- } \\
\text { thermo- } \\
\text { karst }\end{array}$ & 6.23 & 3.00 & $\mathrm{n} / \mathrm{a}$ & 0.41 & 0.127 \\
\hline $\begin{array}{l}\text { Matacha- } \\
\text { rak }\end{array}$ & USA & $\begin{array}{l}\text { Non- } \\
\text { thermo- } \\
\text { karst }\end{array}$ & 2.33 & 1.00 & pos & 0.83 & 0.002 \\
\hline MB-AC & Canada & Hillslope & 12.56 & 2.56 & pos & 0.84 & $<0.0001$ \\
\hline MB-S & Canada & Hillslope & 6.77 & 0.40 & $\mathrm{n} / \mathrm{a}$ & 0.00 & 1 \\
\hline Mcleod & USA & Hillslope & 2.65 & 0.70 & pos & 0.48 & 0.006 \\
\hline North & Canada & Hillslope & 1.35 & 0.53 & pos & 0.82 & 0.0003 \\
\hline Nunatak & $\begin{array}{c}\text { Green- } \\
\text { land }\end{array}$ & $\begin{array}{l}\text { Non- } \\
\text { thermo- } \\
\text { karst }\end{array}$ & 8.24 & 1.62 & pos & 0.81 & 0.016 \\
\hline $\begin{array}{l}\text { Ossian } \\
\text { Sarsfjel- } \\
\text { let }\end{array}$ & Norway & $\begin{array}{l}\text { Non- } \\
\text { thermo- } \\
\text { karst }\end{array}$ & 4.00 & 1.86 & pos & 0.72 & 0.009 \\
\hline Perfect & USA & Hillslope & 5.85 & 1.61 & pos & 0.88 & $<0.0001$ \\
\hline Relaxing & USA & Hillslope & 2.48 & 0.99 & pos & 0.85 & $<0.0001$ \\
\hline $\begin{array}{l}\text { Rocky } \\
\text { Basin }\end{array}$ & Canada & $\begin{array}{l}\text { Non- } \\
\text { thermo- } \\
\text { karst }\end{array}$ & 2.35 & 1.00 & pos & 1.00 & 0.03 \\
\hline Romulus & Canada & Hillslope & 7.77 & 2.65 & pos & 0.78 & 0.002 \\
\hline
\end{tabular}

\begin{tabular}{|c|c|c|c|c|c|c|c|}
\hline Lake & Country & $\begin{array}{l}\text { Landscape } \\
\text { type }\end{array}$ & $\begin{array}{l}\text { Mean } \mathrm{Hg} \\
\text { Accum. } \\
\left(\mu \mathrm{g} / \mathrm{m}^{2} /\right. \\
\text { year) }\end{array}$ & $\sigma$ & Trend & $\tau$ & $\mathrm{p}$ \\
\hline Rummy & Canada & Hillslope & 6.80 & 0.94 & $\mathrm{n} / \mathrm{a}$ & 0.62 & 0.072 \\
\hline SHI-L4 & Canada & $\begin{array}{l}\text { Non- } \\
\text { thermo- } \\
\text { karst }\end{array}$ & 6.46 & 3.03 & pos & 0.82 & $<0.0001$ \\
\hline SHI-L7 & Canada & $\begin{array}{l}\text { Non- } \\
\text { thermo- } \\
\text { karst }\end{array}$ & 20.34 & 5.61 & $\mathrm{n} / \mathrm{a}$ & 0.17 & 0.602 \\
\hline Surprise & USA & Hillslope & 3.83 & 2.10 & pos & 0.90 & $<0.0001$ \\
\hline Vassauga & Norway & $\begin{array}{l}\text { Non- } \\
\text { thermo- } \\
\text { karst }\end{array}$ & 7.30 & 3.35 & pos & 0.93 & 0.002 \\
\hline West & Canada & hillslope & 8.21 & 4.13 & pos & 0.76 & 0.006 \\
\hline Wonder & USA & Hillslope & 2.59 & 1.26 & pos & 0.93 & 0.002 \\
\hline Yterjørna & Norway & $\begin{array}{l}\text { Non- } \\
\text { thermo- } \\
\text { karst }\end{array}$ & 12.21 & 9.69 & pos & 0.84 & $<0.0001$ \\
\hline
\end{tabular}

Page 7, Results, under heading Historical changes in lake sediment mercury and primary production, lines $2-4$ : The following sentence, which previously read:

"The bottom of the core was dated to 1942, suggesting a relatively high rate of sediment accumulation (mean $0.10 \pm 0.06 \mathrm{~g} / \mathrm{cm}^{2} /$ year)."

Should read:

"The bottom of the core was dated to 1942, suggesting a relatively high rate of sediment accumulation (mean $0.042 \pm 0.023 \mathrm{~g} / \mathrm{cm}^{2} /$ year $) . "$

Page 7, Results, under heading Historical changes in lake sediment mercury and primary production, lines $12-14$ : The following sentence, which previously read:

"Mercury accumulation rates were variable (mean $=91.96 \pm 36.1 \mu \mathrm{g} / \mathrm{m}^{2} /$ year), and no significant monotonic trend was detected"

Should read:

"Mercury accumulation rates were variable (mean $=37.40 \pm 14.69 \mu \mathrm{g} / \mathrm{m}^{2} /$ year), and no significant monotonic trend was detected"

Page 8, left column, paragraph 1, lines 4-5: The following sentence, which previously read:

"Sedimentation rate was lower than that observed in BRW100 (mean $0.03 \pm 0.01 \mathrm{~g} / \mathrm{cm}^{2} /$ year)."

Should read:

"Sedimentation rate was lower than that observed in BRW100 (mean $0.014 \pm 0.0061 \mathrm{~g} / \mathrm{cm}^{2} /$ year)."

Page 8, left column, paragraph 1, lines 14-17: The following sentence, which previously read:

"Mercury accumulation rates (mean $14.2 \pm 3.6 \mu \mathrm{g} / \mathrm{m}^{2} /$ year) increased significantly between the 1880s and 2014, more than doubling from 7.0 to $15 \mu \mathrm{g} / \mathrm{m}^{2} /$ year (Fig. 3; Mann-Kendall: $S=107, \tau=-0.42, p=0.04)$."

Should read: 
"Mercury accumulation rates (mean $6.46 \pm 1.64 \mu \mathrm{g}$ / $\mathrm{m}^{2} /$ year) increased significantly between the $1880 \mathrm{~s}$ and 2014, more than doubling from 3 to $7 \mu \mathrm{g} / \mathrm{m}^{2} /$ year (Fig. 3; Mann-Kendall: $S=107, \tau=-0.42, p=0.04) . "$

Page 8 , right column, paragraph 2 , lines $2-4$ : The following sentence, which previously read:

"accumulation rate (mean $0.13 \pm 0.12 \mathrm{~g} / \mathrm{m}^{2} /$ year) of similar magnitude to BRW 100 and $>$ fourfold higher than ATQ206."

Should read:

"accumulation rate (mean $0.08 \pm 0.07 \mathrm{~g} / \mathrm{m}^{2} /$ year) of similar magnitude to BRW 100 and $>$ fourfold higher than ATQ206."

Page 8, right column, paragraph 2, lines 10-12: The following sentence, which previously read:

"Mean mercury accumulation rate $\left(78.6 \pm 68.5 \mu \mathrm{g} / \mathrm{m}^{2} /\right.$ year) was more similar to BRW100 $\left(91.96 \mu \mathrm{g} / \mathrm{m}^{2} /\right.$ year $)$ than to ATQ206 (mean $14.2 \mu \mathrm{g} / \mathrm{m}^{2} /$ year), and similar to BRW100,"

Should read:

"Mean mercury accumulation rate $\left(45.84 \pm 40.52 \mu \mathrm{g} / \mathrm{m}^{2} /\right.$ year) was more similar to BRW100 $\left(37.40 \pm 14.69 \mu \mathrm{g} / \mathrm{m}^{2} /\right.$ year) than to ATQ206 (mean $6.46 \pm 1.64 \mu \mathrm{g} / \mathrm{m}^{2} /$ year), and similar to BRW100,"

Page 10, right column, under heading Comparison of mercury accumulation with other Arctic and subarctic lakes, lines 1-22: The following sentence, which previously read:

"Of the eleven additional Alaskan lakes for which data were available, the majority (8) had mean $\mathrm{Hg}$ accumulation rates that were most similar to that observed for ATQ206 $\left(14.2 \pm 3.60 \mu \mathrm{g} / \mathrm{m}^{2} /\right.$ year); that is, much lower (mean $\mathrm{Hg}$ accumulation $=11.2 \pm 8.8 \mu \mathrm{g} / \mathrm{m}^{2} /$ year) and more uniform (mean temporal standard deviation $=4.2 \pm 4.7$ ) than what we observed in either BRW100 $\left(92.0 \pm 36.1 \mu \mathrm{g} / \mathrm{m}^{2} /\right.$ year $)$ or RDC312 $\left(78.6 \pm 69.5 \mu \mathrm{g} / \mathrm{m}^{2} /\right.$ year; Table 2$)$. When the additional 33 Arctic lakes with available data, including the seven aforementioned Alaskan lakes, and lakes from Canada, Greenland, and Norway (Fig. 1; Table 2) were separated by landscape type (lake thermokarst, hillslope thermokarst, and non-thermokarst), significant differences were found in both mean $\mathrm{Hg}$ accumulation (ANOVA, $\mathrm{F}_{2,37}=3.66, \mathrm{p}=0.036$ ) and temporal variability (standard error, ANOVA, $F_{2,37}=16.64, p<0.0001$; Fig. 6). Post-hoc Tukey's tests indicated that lakes in lake thermokarst landscapes had significantly higher mean $\mathrm{Hg}$ accumulation than lakes in non-thermokarst landscapes $(\mathrm{p}=0.03)$, and that $\mathrm{Hg}$ accumulation in lakes from lake thermokarst landscapes was significantly more temporally variable than that in hillslope thermokarst landscapes $(<0.0001)$ or non-thermokarst landscapes $(\mathrm{p}<0.0001)$.

Should read:

"Of the eleven additional Alaskan lakes for which data were available, the majority (8) had mean $\mathrm{Hg}$ accumulation rates that were most similar to that observed for ATQ206 $\left(6.46 \pm 1.64 \mu \mathrm{g} / \mathrm{m}^{2} /\right.$ year); that is, much lower (mean $\mathrm{Hg}$ accumulation $=4.83 \pm 3.33 \mu \mathrm{g} / \mathrm{m}^{2} /$ year) and more uniform (mean temporal standard deviation $=1.38 \pm 0.55$ ) than what we observed in either BRW100 (37.40 $\pm 14.69 \mu \mathrm{g} / \mathrm{m}^{2} /$ year $)$ or RDC312 $\left(45.84 \pm 40.52 \mu \mathrm{g} / \mathrm{m}^{2} /\right.$ year; Table 2$)$. When the additional 33 Arctic lakes with available data, including the seven aforementioned Alaskan lakes, and lakes from Canada, Greenland, and Norway (Fig. 1; Table 2) were separated by landscape type (lake thermokarst, hillslope thermokarst, and non-thermokarst), significant differences were found in both mean $\mathrm{Hg}$ accumulation (ANOVA, $\mathrm{F}_{2,37}=17.64, \mathrm{p}<0.0001$ ) and temporal variability (standard error, ANOVA, $\mathrm{F}_{2,37}=18.83, \mathrm{p}<0.0001$; Fig. 6). Post-hoc Tukey's tests indicated that lakes in lake thermokarst landscapes had significantly higher mean $\mathrm{Hg}$ accumulation than lakes in non-thermokarst and hillslope thermokarst landscapes $(\mathrm{p}<0.01)$, and that $\mathrm{Hg}$ accumulation in lakes from lake thermokarst landscapes was significantly more temporally variable than that in hillslope thermokarst landscapes $(<0.0001)$ or non-thermokarst landscapes $(\mathrm{p}<0.0001)$.

Figure captions 2, 3 and 4: Previously read:

Fig. 2 Temporal profiles of sediment mercury concentration, accumulation rate, sedimentation rate, percent organic matter, percent mineral matter, and VRS-inferred chlorophyll $a$ for lake BRW100 on the Arctic Coastal Plain of Alaska sampled in August 2014

Fig. 3 Temporal profiles of sediment mercury concentration, accumulation rate, sedimentation rate, percent organic matter, percent mineral matter, and VRS-inferred chlorophyll $a$ for lake ATQ206 on the Arctic Coastal Plain of Alaska sampled in August 2014

Fig. 4 Temporal profiles of sediment mercury concentration, accumulation rate, sedimentation rate, percent organic matter, percent mineral matter, and VRS-inferred chlorophyll $a$ for lake RDC312 on the Arctic Coastal Plain of Alaska sampled in August 2014

should read:

Fig. 2 Temporal profiles of sediment mercury concentration, accumulation rate, sedimentation rate, percent organic matter, percent mineral matter, and VRS-inferred chlorophyll $a$ for lake BRW 100 on the Arctic Coastal Plain of Alaska sampled in August 2014. The scale changes on the $\mathrm{Hg}$ Accumulation (range $=22.9-72.5$ ) and Sedimentation Rate (range $=0.02-0.15$ ) panels of this figure

Fig. 3 Temporal profiles of sediment mercury concentration, accumulation rate, sedimentation rate, percent organic matter, percent mineral matter, and VRS-inferred chlorophyll a for lake ATQ206 on the Arctic Coastal Plain of Alaska sampled in August 2014. The scale changes on the Hg Accumulation (range $=2.7-9.8$ ) and Sedimentation Rate (range $=0.008-0.04)$ panels of this figure 
Fig. 4 Temporal profiles of sediment mercury concentration, accumulation rate, sedimentation rate, percent organic matter, percent mineral matter, and VRS-inferred chlorophyll a for lake RDC312 on the Arctic Coastal Plain of Alaska sampled in August 2014. The scale changes on the $\mathrm{Hg}$ Accumulation (range $=20.5-145.9$ ) and Sedimentation Rate (range $=0.04-0.33$ ) panels of this figure

Figure 6: Previously read:

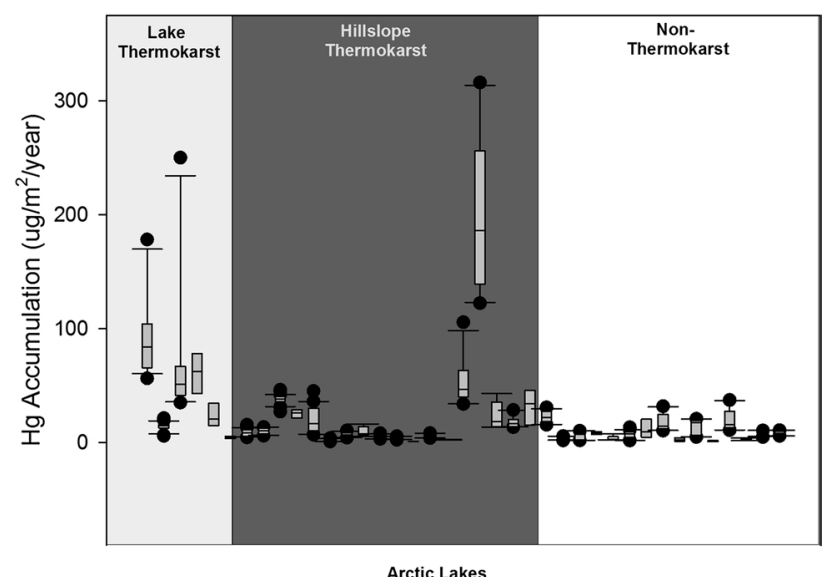

Fig. 6 Box and whisker plots for mercury accumulation rates for 37 circumarctic and 4 subarctic Alaskan lakes on different landscape types, as defined in Olefeldt et (2016). The range bars are 95\% confidence intervals; boxes show the inter-quartile ranges (25-75\%), the horizontal line indicates the median. The lakes in 'lake thermokarst' panel represent, in order: BRW100, ATQ206, and RDC312 (this study), and lakes 2A, and 2B (data from Deison et al. 2012). Accumulation rates are significantly higher and more variable in Lake Thermokarst lakes than Non-thermokarst lakes. Accumulation rates are focus-corrected when data were available (see Online Resource 1)
Should read:

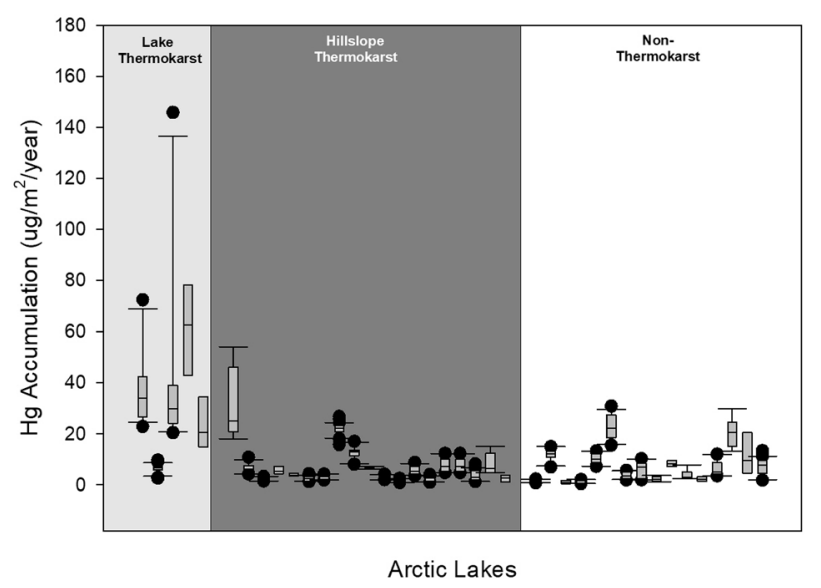

Fig. 6 Box and whisker plots for mercury accumulation rates for 36 circumarctic and 4 subarctic Alaskan lakes on different landscape types, as defined in Olefeldt et (2016). The range bars 95\% confidence intervals; boxes show the inter-quartile ranges (25\%-75\%), the horizontal line indicates the median. The lakes in 'lake thermokarst' panel represent, in order: BRW 100, ATQ 206, and RDC 312 (this study), and lakes 2A, and 2B (data from Deison et al. 2012). Accumulation rates are significantly higher and more variable in Lake Thermokarst lakes than Non-thermokarst lakes and hillslope thermokarst lakes. Accumulation rates are focus-corrected when data were available (see Online Resource)

Publisher's Note Springer Nature remains neutral with regard to jurisdictional claims in published maps and institutional affiliations. 